\title{
COMPARAÇÃO DOS MÉTODOS DE DETERMINAÇÃO DA ESTABILIDADE OXIDATIVA DE BIODIESEL B100, EM MISTURA COM ANTIOXIDANTES SINTÉTICOS. APLICAÇÃO DO DELINEAMENTO SIMPLEX- CENTROIDE COM VARIÁVEL DE PROCESSO
}

\author{
João Rafael de Moraes Cini, Dionísio Borsato*, Carmen Luísa Barbosa Guedes, Hágata Cremasco da Silva e Rodolfo \\ Lopes Coppo \\ Departamento de Química, Universidade Estadual de Londrina, CP 6001, 86051-990 Londrina - PR, Brasil
}

Recebido em 12/4/12; aceito em 14/8/12; publicado na web em 27/11/12

\begin{abstract}
COMPARISON OF METHODS FOR DETERMINATION OF OXIDATIVE STABILITY OF B100 BIODIESEL MIXED WITH SYNTHETIC ANTIOXIDANTS. APPLICATION OF SIMPLEX-CENTROID DESIGN WITH PROCESS VARIABLE. The Rancimat and accelerated stove tests were used to determine the oxidative stability of B100 biodiesel mixed with synthetic antioxidants. The predictive equations, with process variable, were obtained by applying a simplex-centroid design. Regardless of the antioxidant used, all assays carried out with the accelerated stove test presented storage time longer than $177.88 \mathrm{~d}$, the greatest value obtained by applying the Rancimat test. The t test, applied to the parameters containing the process variable, showed a statistically significant difference (at the level of 5\%) between the methods used.
\end{abstract}

Keywords: biodiesel; process variable; mixture design.

\section{INTRODUÇÃO}

O Brasil é um país mundialmente conhecido na área dos biocombustíveis, porque foi pioneiro no uso de álcool como combustível automotor, o que deu ao país um lugar de destaque nesse cenário dos combustíveis renováveis, que a cada dia se torna um mercado tecnológico muito cobiçado. Hoje também se destaca o biodiesel, que apesar de fornecer em torno de $10 \%$ menos energia que o diesel de petróleo, tem um desempenho no motor, no que diz respeito à potência e ao torque, muito próximo ao combustível fóssil, com a vantagem de apresentar maior viscosidade proporcionando melhor lubrificação que o diesel mineral, resultando em um menor desgaste das partes móveis do motor. Além disso, o biodiesel apresenta maior eficiência na queima, diminuindo substancialmente a deposição de resíduos nas partes internas do motor. ${ }^{1}$

A produção do biodiesel ocorre por meio de reações de transesterificação de óleos vegetais, em meio ácido ou básico, onde um triglicerídeo e um álcool, na presença de um catalisador, formam ésteres e glicerol como subproduto. ${ }^{2}$

Borsato et al. ${ }^{3}$ obtiveram biodiesel por meio da transesterificação de óleo de soja, aplicando o delineamento experimental de mistura simplex-centroide. Os resultados da otimização indicaram que o melhor rendimento da reação foi alcançado quando se utilizou o metanol e uma mistura de catalisadores contendo $30,77 \%$ de $\mathrm{NaOH}$ e 69,23\% de metóxido de sódio.

O desenvolvimento de qualquer produto envolvendo mais de um componente requer algumas formas particulares de experimentos de mistura. ${ }^{4}$ Delineamentos de experimentos são uma base de conhecimento fundamentada em estatística e outras disciplinas científicas, para planejamentos eficientes e efetivos de experimentos e para fazer inferências significativas sobre os dados experimentais. Atualmente, o delineamento de experimentos é visto como uma tecnologia de qualidade para se atingir a excelência de um produto, sendo um instrumento usado para otimizar sistemas e processos, acelerar o ciclo de desenvolvimento, reduzir os custos e solucionar problemas de fabricação. Dentre os diversos tipos de delineamentos destaca-se

*e-mail: dborsato@uel.br o de misturas, que tem sido utilizado por diversos pesquisadores com o objetivo de desenvolver novos produtos. ${ }^{5,6}$ Nestes ensaios, dois ou mais componentes são misturados em várias proporções e as características dos produtos resultantes são registradas. As respostas dependem, somente, das proporções dos componentes presentes na mistura e não da sua quantidade absoluta..$^{5,7-9}$

Em adição ao trabalho de Scheffé, ${ }^{7}$ outros têm sido apresentados utilizando os delineamentos de misturas. Mais recentemente, Borsato et al. ${ }^{10}$ apresentaram uma análise da cinética da oxidação de biodiesel B100 aplicando o delineamento simplex-centroide. Maia et al., ${ }^{11}$ utilizando o mesmo delineamento, estudaram a eficiência de antioxidantes sintéticos, quando adicionados ao biodiesel B100 derivado de óleo de soja.

Além dos componentes de misturas, alguns tipos de problemas envolvem variáveis de processo. Fatores como $\mathrm{pH}$, temperatura e concentrações dos reagentes são exemplos típicos de variáveis de processo. Alterações nos níveis dessas variáveis podem afetar o resultado experimental através de seus efeitos individuais e de interação com outras variáveis de mistura, mas seus níveis podem ser variados de forma independente. ${ }^{12}$ Scheffé 7 e Hare ${ }^{13}$ apresentaram trabalhos envolvendo delineamentos de misturas com variáveis de processo. Cornell e Deng ${ }^{8}$ combinaram variáveis de processo com ingredientes de misturas em experimentos na área de alimentos. Anderson-Cook et al. ${ }^{14}$ realizaram experimentos envolvendo componentes de mistura com variáveis de processos na formulação de produtos farmacêuticos.

Além dos delineamentos experimentais, os métodos de otimização têm sido aplicados de maneira variada na indústria de processos químicos, térmicos e gerenciamento para redução de custos. ${ }^{15} \mathrm{Em}$ relação à obtenção de formulações ótimas, os delineamentos de mistura podem gerar várias respostas simultaneamente, o que pode dificultar a busca da solução ótima. ${ }^{15,16}$ Derringer e Suich $^{17}$ desenvolveram um método para determinar a melhor combinação de múltiplas respostas com restrições. Eles sugeriram uma função de compromisso, que define os intervalos desejáveis. Essa abordagem é considerada um método de programação não linear para uma solução ótima a partir de uma combinação simultânea de modelos com restrições.

O objetivo do presente trabalho foi comparar os métodos de determinação da estabilidade oxidativa de biodiesel B100, por Rancimat 
e teste acelerado em estufa, em mistura com antioxidantes sintéticos, utilizando delineamento experimental de misturas incluindo a variável de processo.

\section{PARTE EXPERIMENTAL}

\section{Biodiesel}

Utilizou-se Biodiesel B100 obtido por transesterificação de óleo de soja por rota metílica, utilizando-se o metóxido de sódio como catalisador. $^{3}$

\section{Análise cromatográfica}

Foi utilizado o cromatógrafo a gás modelo GC-17A com detector de ionização de chama (Shimadzu), com coluna DB1 (J\&W Scientific) $-100 \%$ polimetilsiloxano com $30 \mathrm{~m}$ de comprimento $\mathrm{x} 0,25 \mathrm{~mm}$ de d.i. $\mathrm{x} 0,25 \mu \mathrm{m}$ de espessura de filme. As temperaturas do injetor split e do detector foram mantidas a $340^{\circ} \mathrm{C}$. A rampa de aquecimento da coluna foi mantida inicialmente a $50^{\circ} \mathrm{C}$ por 2 min e, na seqüência, aquecida à razão de $10^{\circ} \mathrm{C}$ min $^{-1}$ até $180^{\circ} \mathrm{C}$ mantendo-a nessa temperatura por 2 min; a seguir, aquecida à razão de $15^{\circ} \mathrm{C} \mathrm{min}{ }^{-1}$ até $340^{\circ} \mathrm{C}$, permanecendo nessa temperatura por $10 \mathrm{~min}$. A vazão do gás de arraste, $\mathrm{N}_{2}$, foi de 1,5 $\mathrm{mL} \min ^{-1}$ e o volume de injeção de $2,0 \mu \mathrm{L}$ com razão de split 10 . Os dados foram coletados por meio do software CLASS-CR10 Shimadzu.

\section{Antioxidantes}

Os antioxidantes grau P.A. butil-hidroxianisol (BHA) Synth, butil-hidroxitolueno (BHT) Synth e terc-butil-hidroquinona (TBHQ) Acros, cujas proporções foram estabelecidas pelo delineamento de mistura, foram adicionados diretamente ao biodiesel B100 antes da avaliação da estabilidade oxidativa. A concentração total de antioxidante adicionado foi de $6,0 \times 10^{-3} \mathrm{~mol} \mathrm{~L}^{-1}$, aproximadamente $0,1 \%$ $(\mathrm{m} / \mathrm{v})$. Este valor teve como base ensaios preliminares.

\section{Avaliação da estabilidade oxidativa determinada pelo teste Rancimat}

As amostras de $3 \mathrm{~g}$ de biodiesel, valor estabelecido pela norma EN14112, ${ }^{18}$ contendo as quantidades de antioxidantes estabelecidas pelo delineamento simplex-centroide, bem como as amostras controle, foram levadas ao aquecimento acelerado a $100,110,120$ e $130^{\circ} \mathrm{C}$, com taxa de insuflação de ar de $10 \mathrm{~L} \mathrm{~h}^{-1}$, para determinação do período de indução. As amostras do controle e do ponto central foram realizadas em triplicatas e nos demais pontos em amostra única. $\mathrm{O}$ teste foi efetuado utilizando o Rancimat 873, em concordância com a norma oficial de determinação da estabilidade oxidativa em teste acelerado. ${ }^{18}$

\section{Avaliação da estabilidade oxidativa determinada pelo teste de estufa}

As amostras de $30 \mathrm{~g}$ de biodiesel, quantidade suficiente para realizar os ensaios, contendo as quantidades de antioxidantes estabelecidas pelo delineamento simplex-centroide, bem como as amostras controle, foram levadas ao aquecimento acelerado em estufa a 30, 50 e $80{ }^{\circ} \mathrm{C}$ durante o tempo necessário para que o maior índice de peróxido ${ }^{19-21}$ de cada amostra pudesse ser determinado.

\section{Período de indução}

Corresponde ao valor do ponto de inflexão (derivada $2^{\mathrm{a}}$ ) da curva obtida pelo índice de peróxido versus tempo para o método de estufa.

\section{Índice de peróxido (I.P.)}

Foi determinado por titulação com tiossulfato de sódio $0,1 \mathrm{~N}$, segundo a AOAC..$^{22}$

\section{Delineamento experimental para mistura ${ }^{23}$}

Foi utilizado o planejamento simplex-centroide, com duas repetições no ponto central, ${ }^{6}$ com $2^{4}-1$ combinações de misturas, sendo q o número de componentes com soma igual a 1 ou $100 \% .{ }^{24}$

\section{Modelo matemático para o delineamento de misturas}

A função utilizada foi do tipo

$Y(\mathrm{x})=\sum_{1 \leq i \leq q}^{q} \beta_{i} \mathrm{x}_{i}+\sum_{1 \leq i \leq j \leq q}^{q} \beta_{i j} \mathrm{x}_{i} \mathrm{x}_{j}+\beta_{123} \mathrm{x}_{1} \mathrm{x}_{2} \mathrm{x}_{3}$

onde, $\mathrm{Y}$ representa a função resposta dos dados experimentais; $\mathrm{x}_{1}, \mathrm{x}_{2}$ e $\mathrm{x}_{3}$ são as variáveis independentes e correspondem à porcentagem de BHA, BHT e TBHQ na mistura, respectivamente, e $\beta$ os parâmetros estimados. ${ }^{5,23}$

\section{Modelo matemático combinado}

A função 2 representa o modelo combinado, contendo as variáveis independentes, representando os três antioxidantes utilizados e a variável discreta de processo, que representa o método utilizado para a determinação do período de indução. O modelo foi ajustado para a combinação $\left(2^{\mathrm{q}}-1\right) \times 2^{\mathrm{n}}$ onde $\mathrm{n}$ representa o número de variáveis discretas de processo, representada na Equação 2 por z, que foi codificada para $\mathrm{z}=+1$, para os dados obtidos pelo teste Rancimat, e $\mathrm{z}=-1$, para os dados obtidos pelo teste acelerado em estufa. Os coeficientes de regressão do modelo foram obtidos, pelo método dos mínimos quadrados, através da equação matricial $\gamma=\left(X^{\prime} X\right)^{-1} X^{\prime} Y$, onde $X$ é a matriz do delineamento incluindo a variável de processo e $\mathrm{Y}$ é o vetor resposta.

$$
\begin{aligned}
Y(\mathrm{x}, \mathrm{z})= & \sum_{1 \leq i \leq q} \gamma_{i}^{\circ} \mathrm{x}_{i}+\sum_{1 \leq i \leq q} \gamma_{i}^{1} \mathrm{x}_{i} z+\sum_{1 \leq i \leq j \leq q} \gamma_{i j}^{\circ} \mathrm{x}_{i} \mathrm{x}_{j}+ \\
& \sum_{1 \leq i \leq j \leq q} \gamma_{i j}^{1} \mathrm{x}_{i} \mathrm{x}_{j} z+\gamma_{123}^{\circ} \mathrm{x}_{1} \mathrm{x}_{2} \mathrm{x}_{3}+\gamma_{123}^{1} \mathrm{x}_{1} \mathrm{x}_{2} \mathrm{x}_{3} z
\end{aligned}
$$

Na Equação 2, $\gamma^{\circ}$ representa os parâmetros estimados para os termos sem a variável de processo e $\gamma^{1}$ aqueles estimados para os termos contendo a variável de processo. ${ }^{5,13}$

\section{Análise estatística}

Os coeficientes da regressão foram estimados utilizando o software Statistica v.9.0. ${ }^{23}$

\section{Análise de conformidade}

A massa específica do biodiesel B100 foi determinada segundo o método ASTM D4052, ${ }^{25}$ o ponto de fulgor segundo ASTM D93, ${ }^{26} \mathrm{O}$ índice de acidez pela ASTM D664, ${ }^{27}$ teor de glicerina livre e total pelo método ASTM $6584,{ }^{28}$ mono, di e tri glicerídeos pela norma ASTM $6584,{ }^{28}$ teor de álcool pela EN $14110^{29}$ e de ésteres pela EN $14103 .{ }^{30}$

\section{RESULTADOS E DISCUSSÃO}

\section{Caracterização do biodiesel B100 de óleo de soja}

O teor de ésteres metílicos no biodiesel é um parâmetro previsto na norma EN $14214^{31}$ cuja porcentagem mínima exigida de éster é de 
96,5\% (massa), a ser determinada através do método cromatográfico EN $14103 .^{30}$

A análise cromatográfica mostrou que o biodiesel utilizado consistia basicamente dos ésteres etil palmítico, etil oleato, etil linoleato, etil linoleneato, bem como outros etil ésteres cuja composição varia do C14:0 até C24:0. Os ésteres com composição química variando de C8 a C14, respectivamente, representaram $0,33 \%(\mathrm{~m} / \mathrm{m})$ da amostra do biodiesel utilizado. O teor de éster metílico do ácido graxo C16:0 foi de 14,81\% e dos ésteres metílicos C18:0; C18:1 e C18:2 foi de $80,10 \%(\mathrm{~m} / \mathrm{m})$. Os ésteres formados por C20 a C22 representaram $2,04 \%(\mathrm{~m} / \mathrm{m})$ da amostra do biodiesel. Portanto, estes ésteres juntos somam $97,28 \%(\mathrm{~m} / \mathrm{m})$ das substâncias presentes no biodiesel B100 utilizado, estando em acordo com as especificações estabelecidas pela União Europeia e pela Agência Nacional do Petróleo, Gás Natural e Biocombustíveis (ANP), sendo que os teores de ácidos graxos livres, álcool, glicerina e água devem ser mínimos, de modo que a pureza do biodiesel deve ser maior que $96,5 \%(\mathrm{~m} / \mathrm{m}) .{ }^{32,33}$ Além disso, os teores de mono, di e triglicerídeos presentes somaram juntos $0,35 \%$ e o teor de glicerina livre verificado foi de $0,002 \%(\mathrm{~m} / \mathrm{m})$, valor bastante inferior ao da legislação brasileira, que prevê um teor máximo de glicerina livre de $0,02 \%(\mathrm{~m} / \mathrm{m}){ }^{33}$

A determinação da glicerina residual serve como parâmetro para se avaliar a eficiência do processo de purificação do biodiesel. Altas concentrações de glicerina no biodiesel provocam problemas de armazenamento, pois quando ele é misturado com o diesel de petróleo se observa a separação da glicerina nos tanques de estocagem. Problemas como formação de depósitos, entupimento dos bicos injetores do motor e emissões de aldeídos também estão relacionados com a alta concentração da glicerina no biodiesel. ${ }^{1}$

Para assegurar a qualidade do biodiesel é necessário estabelecer padrões de qualidade, objetivando fixar teores limites dos contaminantes que não venham prejudicar a qualidade das emissões da queima, bem como o desempenho, a integridade do motor e a segurança no transporte e manuseio. Devem ser monitoradas também possíveis degradações do produto durante o processo de estocagem. ${ }^{1}$

A Tabela 1S, material suplementar, contendo os principais parâmetros de conformidade do biodiesel B100 utilizado, mostra

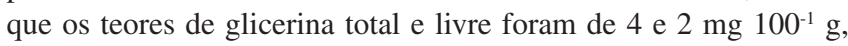
respectivamente, valores bastante inferiores aos da legislação brasileira, que prevê um teor máximo de glicerina total de $0,25 \%(\mathrm{~m} / \mathrm{m}) \mathrm{e}$ livre de $0,02 \%(\mathrm{~m} / \mathrm{m}) .{ }^{33}$ Além disso, os valores obtidos para a massa específica, ponto de fulgor, índice de acidez e teor de metanol estão de acordo com a legislação vigente. ${ }^{33}$

\section{Estabilidade oxidativa determinada pelo teste Rancimat}

Para se avaliar a estabilidade oxidativa ou sua susceptibilidade à oxidação, o biodiesel B100, acrescentado de antioxidantes, foi submetido ao teste de oxidação acelerado. ${ }^{18}$

O delineamento experimental de mistura simplex-centroide, composto de 7 ensaios com 2 repetições no ponto central (Tabela 1), foi utilizado para avaliar o efeito da adição dos antioxidantes BHA $\left(\mathrm{x}_{1}\right)$, BHT $\left(\mathrm{x}_{2}\right)$ e TBHQ $\left(\mathrm{x}_{3}\right)$ em biodiesel B100 obtido do óleo de soja.

A especificação estabelecida pelo método de ensaio EN $14112^{18}$ indica que o período de indução mínimo deve ser superior a $6 \mathrm{~h}$, a $110{ }^{\circ} \mathrm{C}$. De acordo com a Tabela 1, para esta temperatura, todos os ensaios exceto o número 4 , uma das repetições do ponto central e o controle apresentaram o período de indução menor que $6 \mathrm{~h}$. O período de indução de $3,28 \mathrm{~h}$ para o controle apresentou valor muito inferior ao mínimo estabelecido, para $110^{\circ} \mathrm{C}$, mostrando a importância da presença dos antioxidantes na conservação do biodiesel B100..$^{33}$

Segundo Frankel, ${ }^{34}$ os métodos de estabilidade oxidativa acelerada apresentam validade restrita, pois o mecanismo de oxidação muda à
Tabela 1. Valores dos períodos de indução, em horas, obtidos pelo Rancimat segundo o delineamento experimental de mistura simplex-centroide

\begin{tabular}{cccccc}
\hline \multirow{2}{*}{ Ensaio } & \multirow{2}{*}{ Mistura* } & \multicolumn{4}{c}{ Período de indução / h } \\
\cline { 3 - 6 } & & $100{ }^{\circ} \mathrm{C}$ & $110{ }^{\circ} \mathrm{C}$ & $120{ }^{\circ} \mathrm{C}$ & $130{ }^{\circ} \mathrm{C}$ \\
\hline 1 & $(1 ; 0 ; 0)$ & 12,38 & 8,06 & 3,54 & 1,54 \\
2 & $(0 ; 1 ; 0)$ & 12,23 & 6,20 & 2,53 & 1,91 \\
3 & $(0 ; 0 ; 1)$ & 11,17 & 9,78 & 3,27 & 1,55 \\
4 & $(1 / 2 ; 1 / 2 ; 0)$ & 11,83 & 4,62 & 3,45 & 1,56 \\
5 & $(1 / 2 ; 0 ; 1 / 2)$ & 14,42 & 7,91 & 2,55 & 2,05 \\
6 & $(0 ; 1 / 2 ; 1 / 2)$ & 8,95 & 7,76 & 2,48 & 1,59 \\
7 & $(1 / 3 ; 1 / 3 ; 1 / 3)$ & 12,12 & 5,85 & 2,78 & 1,57 \\
8 & $(1 / 3 ; 1 / 3 ; 1 / 3)$ & 11,22 & 6,85 & 2,68 & 1,55 \\
9 & $(1 / 3 ; 1 / 3 ; 1 / 3)$ & 11,67 & 6,35 & 2,73 & 1,58 \\
Controle & $(0 ; 0 ; 0)$ & 5,94 & 3,28 & 1,62 & 0,99 \\
\hline
\end{tabular}

$*\left(\%_{\mathrm{BHA}}, \%_{\mathrm{BHT}}, \%_{\text {твнQ }}\right)$

medida que se submete a amostra ao aquecimento, à luz ou ao contato com metais, enquanto que os testes efetuados em temperatura ambiente se aproximam da estocagem real. Porém, por proporcionarem resultados mais rápidos, os métodos acelerados reduzem o tempo de trabalho e o consumo de reagentes. ${ }^{34}$

Hasenhuettl e Wan ${ }^{35}$ encontraram alta correlação linear entre o logaritmo natural do período de indução em função da temperatura, quando estudaram a estabilidade oxidativa de seis diferentes tipos de óleos vegetais, sem o uso de antioxidantes. Xin et al. ${ }^{36}$ verificaram o mesmo comportamento quando estudaram a estabilidade de biodiesel de óleo de girassol, contendo diferentes concentrações do antioxidante galato de propila.

A Figura 1 mostra a temperatura em função do logaritmo natural do período de indução para o biodiesel B100 de óleo de soja estabilizado com antioxidantes sintéticos e controle. Foi observada uma alta correlação linear entre os diferentes tratamentos e o controle, obtendo-se valores superiores a 0,92 .

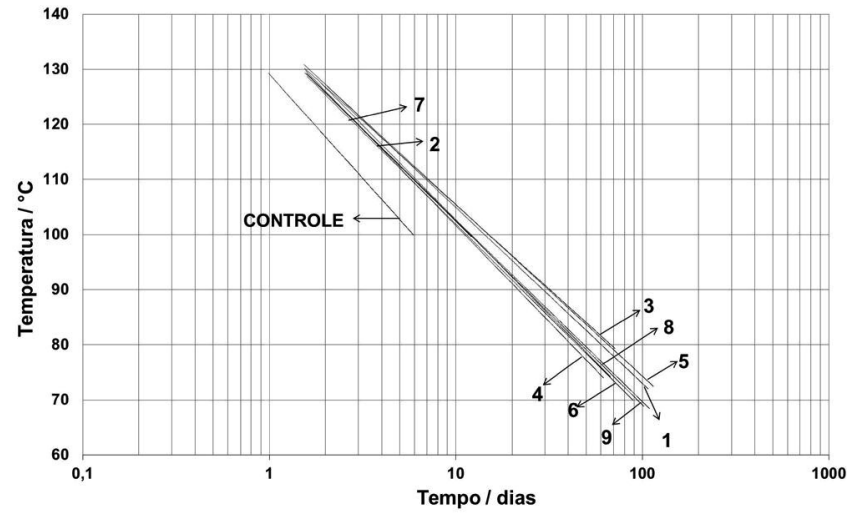

Figura 1. Relação entre o logaritmo natural do período de indução, obtido pelo Rancimat, e a temperatura de ensaio do biodiesel de soja estabilizado com antioxidantes e controle, em dias

Os dados experimentais foram ajustados pelo método dos mínimos quadrados e extrapolados para se obter uma estimativa dos tempos correspondentes ao período de indução para estocagem em temperatura de $25^{\circ} \mathrm{C}$ (Tabela 2). Dessa maneira, verificou-se que os ensaios contendo antioxidantes apresentaram valores superiores aos 23,45 d observados para o controle, com destaque para o tratamento 1, contendo apenas o BHA, com 132,57 d, o tratamento 3 com 177,88 
d, a mistura binária BHA e TBHQ, tratamento 5, com 159,09 d e a mistura ternária, com um valor médio de 89,25 d, ficando os demais com valores inferiores.

Tabela 2. Valores dos períodos de indução em dias, a $25^{\circ} \mathrm{C}$, obtidos segundo o delineamento experimental de mistura simplex-centroide

\begin{tabular}{ccc}
\hline Ensaio & Mistura* & Período de indução / dias \\
\hline 1 & $(1 ; 0 ; 0)$ & 132,57 \\
2 & $(0 ; 1 ; 0)$ & 75,55 \\
3 & $(0 ; 0 ; 1)$ & 177,88 \\
4 & $(1 / 2 ; 1 / 2 ; 0)$ & 65,22 \\
5 & $(1 / 2 ; 0 ; 1 / 2)$ & 159,09 \\
6 & $(0 ; 1 / 2 ; 1 / 2)$ & 80,12 \\
7 & $(1 / 3 ; 1 / 3 ; 1 / 3)$ & 86,84 \\
8 & $(1 / 3 ; 1 / 3 ; 1 / 3)$ & 94,22 \\
9 & $(1 / 3 ; 1 / 3 ; 1 / 3)$ & 86,64 \\
Controle & $(0 ; 0 ; 0)$ & 23,45 \\
\hline
\end{tabular}

$*\left(\%_{\mathrm{BHA}}, \%_{\mathrm{BHT}}, \%_{\mathrm{TBHQ}}\right)$

O domínio experimental consistiu de diferentes proporções dos antioxidantes utilizados, variando de 0 a $100 \%$ sendo $x_{1}$ representado pelo BHA, $x_{2}$ pelo BHT e $x_{3}$ pelo TBHQ. A Equação canônica 3 foi ajustada aos dados experimentais, onde $\mathrm{Y}_{1}$ representa o tempo de estocagem, em dias, quando se utilizou o Rancimat para determinar o período de indução para os três componentes. Os termos com asterisco são significativos em nível de $5 \%$ portanto, o termo de interação entre o BHA e o TBHQ e o termo de interação ternária foram não significativos. Além disso, os termos quadráticos apresentaram coeficientes negativos, indicando que influenciam negativamente o tempo de estocagem do biodiesel B100 de óleo de soja.

$Y_{1}=132,57^{*} x_{1}+75,55^{*} x_{2}+177,88^{*} x_{3}-155,36^{*} x_{1} x_{2}+$

$$
15,46 \mathrm{x}_{1} \mathrm{x}_{3}-186,38^{*} \mathrm{x}_{2} \mathrm{x}_{3}-85,86 \mathrm{x}_{1} \mathrm{x}_{2} \mathrm{x}_{3}
$$

Além dos termos significativos apresentados na Equação 3, a análise de variância mostrou que o modelo é significativo em nível de $5 \%$. O valor do $\mathrm{R}^{2}$ ajustado foi de $98,84 \%$ e o desvio da regressão, desconsiderando o termo de interação binária $\left(\mathrm{x}_{1} \mathrm{x}_{3}\right)$ e o de interação ternária, não significativos, foi de $53,14 \%$ indicando que o modelo pode ser utilizado para fins preditivos.

A região de combinação ternária entre as variáveis independentes $\mathrm{x}_{1}, \mathrm{x}_{2}$ e $\mathrm{x}_{3}$ pode ser observada através das curvas de nível apresentadas na Figura 1S, material suplementar. A Figura $1 \mathrm{~S}$ mostra as regiões de contorno da superfície de resposta para a variável dependente, tempo de estocagem, obtidas pelo modelo matemático, quando se utilizou o Rancimat na determinação do período de indução. Ela também mostra que a melhor estabilidade pode ser alcançada utilizando-se como antioxidante apenas o TBHQ.

A otimização do tempo de estocagem, por meio da equação preditiva, está ilustrada na Figura 2, demonstrando que um tempo de estocagem de 177,88 dias pode ser alcançado quando se usa $100 \%$ de TBHQ.

\section{Estabilidade oxidativa determinada pelo teste acelerado em estufa}

$\mathrm{O}$ delineamento experimental de mistura simplex-centroide, composto de 7 ensaios com 3 repetições no ponto central (Tabela 3), foi utilizado para avaliar o efeito da adição dos antioxidantes

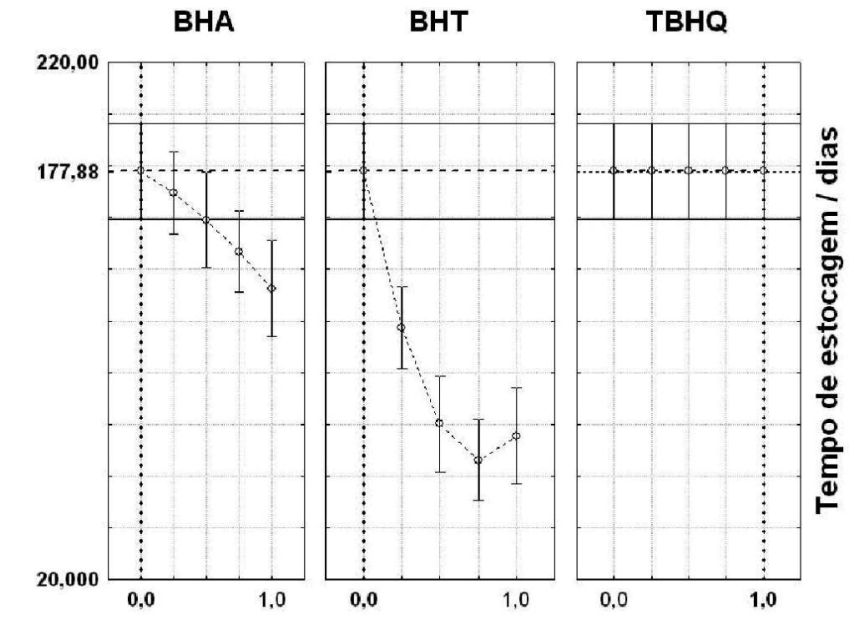

Figura 2. Gráfico das condições ótimas para as variáveis estudadas pelo teste Rancimat

Tabela 3. Valores dos períodos de indução, em horas, obtidos pelo método acelerado em estufa segundo o delineamento experimental de mistura simplex-centroide

\begin{tabular}{ccccc}
\hline \multirow{2}{*}{ Ensaio } & Mistura* & \multicolumn{3}{c}{ Período de indução / h } \\
\cline { 3 - 5 } & & $30{ }^{\circ} \mathrm{C}$ & $50{ }^{\circ} \mathrm{C}$ & $80{ }^{\circ} \mathrm{C}$ \\
\hline 1 & $(1 ; 0 ; 0)$ & 182,35 & 29,78 & 3,75 \\
2 & $(0 ; 1 ; 0)$ & 202,00 & 38,63 & 4,37 \\
3 & $(0 ; 0 ; 1)$ & 182,15 & 50,53 & 4,25 \\
4 & $(1 / 2 ; 1 / 2 ; 0)$ & 186,86 & 22,92 & 4,39 \\
5 & $(1 / 2 ; 0 ; 1 / 2)$ & 196,97 & 51,76 & 4,39 \\
6 & $(0 ; 1 / 2 ; 1 / 2)$ & 100,74 & 60,36 & 3,57 \\
7 & $(1 / 3 ; 1 / 3 ; 1 / 3)$ & 182,16 & 52,00 & 4,31 \\
8 & $(1 / 3 ; 1 / 3 ; 1 / 3)$ & 171,68 & 53,79 & 4,59 \\
9 & $(1 / 3 ; 1 / 3 ; 1 / 3)$ & 182,44 & 52,00 & 4,41 \\
10 & $(1 / 3 ; 1 / 3 ; 1 / 3)$ & 179,74 & 50,60 & 4,44 \\
Controle & $(0 ; 0 ; 0)$ & 75,99 & 18,512 & 4,17 \\
\hline
\end{tabular}

$*\left(\%_{\mathrm{BHA}}, \%_{\mathrm{BHT}}, \%_{\mathrm{TBHQ}}\right)$

BHA $\left(\mathrm{x}_{1}\right)$, BHT $\left(\mathrm{x}_{2}\right)$ e TBHQ $\left(\mathrm{x}_{3}\right)$ em biodiesel B100 obtido do óleo de soja.

A Figura 3 mostra a temperatura em função do logaritmo natural do período de indução para o biodiesel B100 de óleo de soja

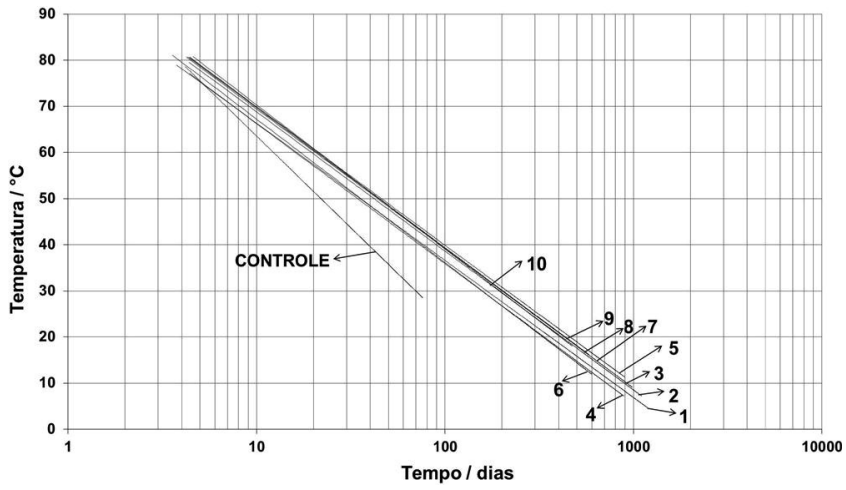

Figura 3. Relação entre o logaritmo natural do período de indução, obtido pelo teste de estufa e a temperatura de ensaio do biodiesel de soja estabilizado com antioxidantes e controle, em dias 
estabilizado com antioxidantes sintéticos e controle. Foi observada uma alta correlação linear entre os diferentes tratamentos e o controle, obtendo-se valores superiores a 0,93 .

Os dados experimentais foram ajustados pelo método dos mínimos quadrados e extrapolados para se obter uma estimativa dos tempos correspondentes ao período de indução para estocagem em temperatura de $25^{\circ} \mathrm{C}$ (Tabela 4). Dessa maneira, verificou-se que os ensaios contendo antioxidantes apresentaram valores superiores aos 94,30 d observados para o controle, com destaque para o tratamento 5 que consiste em uma mistura binária contendo BHA e TBHQ, com 315,64 d, os tratamentos 2 e 3 com 292,94 e 297,79 d, respectivamente, e a mistura ternária, com um valor médio de 296,04 d, ficando os demais tratamentos com períodos de indução inferiores.

Tabela 4. Valores dos períodos de indução em dias, a $25^{\circ} \mathrm{C}$, obtidos segundo o delineamento experimental de mistura simplex-centroide

\begin{tabular}{ccc}
\hline Tratamento & Mistura* & Período de indução / dias \\
\hline 1 & $(1 ; 0 ; 0)$ & 246,21 \\
2 & $(0 ; 1 ; 0)$ & 292,94 \\
3 & $(0 ; 0 ; 1)$ & 297,79 \\
4 & $(1 / 2 ; 1 / 2 ; 0)$ & 234,81 \\
5 & $(1 / 2 ; 0 ; 1 / 2)$ & 315,64 \\
6 & $(0 ; 1 / 2 ; 1 / 2)$ & 227,40 \\
7 & $(1 / 3 ; 1 / 3 ; 1 / 3)$ & 305,09 \\
8 & $(1 / 3 ; 1 / 3 ; 1 / 3)$ & 286,94 \\
9 & $(1 / 3 ; 1 / 3 ; 1 / 3)$ & 300,23 \\
10 & $(1 / 3 ; 1 / 3 ; 1 / 3)$ & 291,91 \\
Controle & $(0 ; 0 ; 0)$ & 94,30 \\
\hline
\end{tabular}

$*\left(\%_{\mathrm{BHA}}, \%_{\mathrm{BHT}}, \%_{\mathrm{TBHQ}}\right)$

O domínio experimental consistiu de diferentes proporções dos antioxidantes utilizados, variando de 0 a $100 \%$, sendo $x_{1}$ representado pelo BHA, $x_{2}$ pelo BHT e $x_{3}$ pelo TBHQ. A Equação canônica 4 foi ajustada aos dados experimentais, onde $Y_{2}$ representa o tempo de estocagem, em dias, quando se utilizou o método de estufa para determinar o período de indução para os três componentes. Os termos com asterisco são significativos em nível de $5 \%$, sendo que as interações entre BHA e BHT e entre BHT e TBHQ apresentaram coeficientes negativos, indicando que influenciam negativamente o tempo de estocagem do biodiesel B100 de óleo de soja.

$$
\begin{aligned}
Y_{2}= & 246,21^{*} x_{1}+292,94^{*} x_{2}+297,79 * x_{3}-139,06 * x_{1} x_{2}+ \\
& 174,56 * x_{1} x_{3}-271,86^{*} x_{2} x_{3}+1172,60 * x_{1} x_{2} x_{3}
\end{aligned}
$$

Além dos termos significativos apresentados na Equação 4, a análise de variância mostrou que o modelo é significativo em nível de $5 \%$ e o valor do $\mathrm{R}^{2}$ ajustado foi de $93,06 \%$, indicando que o modelo pode ser utilizado para fins preditivos apesar do desvio da regressão ser significativo em nível de $1,14 \%$.

A Figura 2S, material suplementar, mostra as regiões de contorno da superfície de resposta para a variável dependente, tempo de estocagem, obtida pelo modelo matemático, quando se utilizou o teste acelerado em estufa na determinação do período de indução. Ela ainda mostra que a melhor estabilidade pode ser alcançada quando se utiliza a mistura de BHA e TBHQ.

A otimização do tempo de estocagem, por meio da equação preditiva, está ilustrada na Figura 4. Ela mostra que um tempo de estocagem de 319,10 dias pode ser alcançado quando se usa $30,77 \%$ de BHA e 69,23\% de TBHQ.

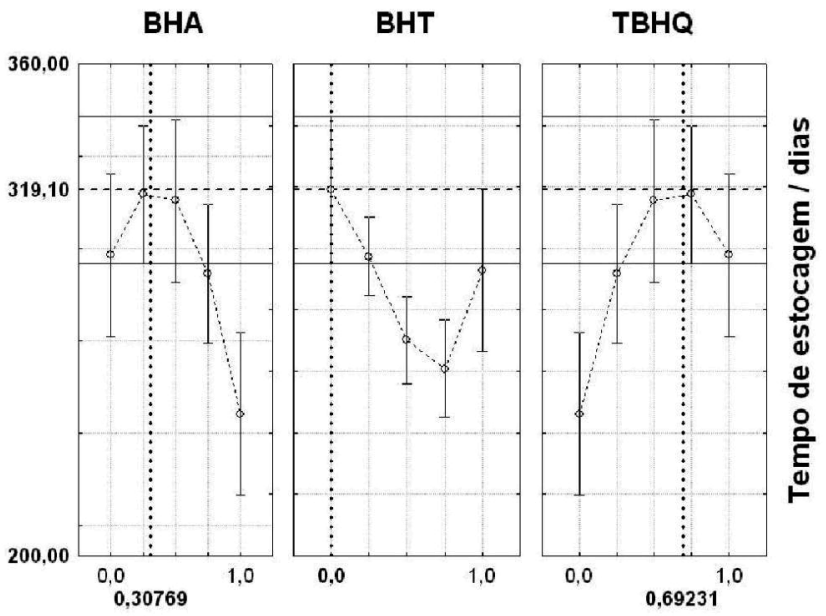

Figura 4. Gráfico das condições ótimas para as variáveis estudadas pelo teste em estufa

\section{Modelo combinado}

O modelo polinomial (Equação 5) foi desenvolvido conforme proposta de Cornell e Deng, ${ }^{8}$ que combinaram os componentes de mistura com variável de processo (Equação 2). Eles mostraram que é possível medir não somente como os componentes de mistura, isolados ou em conjunto, produzem diferentes efeitos nas respostas, mas também como mudanças nas condições de processo podem influenciar o comportamento das misturas desses componentes. As variáveis de processo $(\mathrm{z})$ e de mistura $(x)$ estão presentes nas formulações, e a habilidade de estudar ambos pode conduzir ao melhor entendimento do sistema como um todo. O delineamento de mistura simplex-centroide, no presente estudo, foi associado a uma polinomial, onde o ensaio realizado com o teste acelerado em estufa foi codificado para $\mathrm{z}=-1$ e aquele realizado com o Rancimat foi codificado para $\mathrm{z}=+1$.

$$
\begin{aligned}
\mathrm{Y}(\mathrm{x}, \mathrm{z})= & 189,39 \mathrm{x}_{1}-56,82 \mathrm{x}_{1} \mathrm{z}^{*}+184,25 \mathrm{x}_{2}-108,69 \mathrm{x}_{2} \mathrm{z}^{*}+ \\
& 237,84 \mathrm{x}_{3}-59,96 \mathrm{x}_{3} \mathrm{Z}^{*}-147,21 \mathrm{x}_{1} \mathrm{x}_{2}-8,15 \mathrm{x}_{1} \mathrm{x}_{2} \mathrm{z}+ \\
& 95,01 \mathrm{x}_{1} \mathrm{x}_{3}-79,55 \mathrm{x}_{1} \mathrm{x}_{3} \mathrm{z}^{*}-229,12 \mathrm{x}_{2} \mathrm{x}_{3}+42,74 \mathrm{x}_{2} \mathrm{x}_{3} \mathrm{z}^{*}+ \\
& 542,44 \mathrm{x}_{1} \mathrm{x}_{2} \mathrm{x}_{3}-628,26 \mathrm{x}_{1} \mathrm{x}_{2} \mathrm{x}_{3} \mathrm{z}^{*}
\end{aligned}
$$

Os termos apresentados na Equação 5 foram obtidos a partir da Equação 2, sendo os parâmetros $\gamma^{0}$ determinados tendo como base de cálculo a média aritmética dos parâmetros $\beta$ das Equações 3 e 4 e os parâmetros $\gamma^{1}$ foram obtidos utilizando como base a semidiferença dos parâmetros $\beta$ das mesmas equações.

Aplicando-se o teste t para os parâmetros contendo a variável de processo, verificou-se que todos os termos foram significativos em nível de $5 \%$ de significância, exceto o termo $\left(\mathrm{x}_{1} \mathrm{x}_{2} \mathrm{z}\right)$ que representa uma mistura binária dos antioxidantes BHA e BHT.

A análise da equação mostra que os menores tempos de estocagem, à temperatura de $25^{\circ} \mathrm{C}$, foram obtidos quando se utilizou $\mathrm{o}$ Rancimat na determinação do ponto de inflexão, isto é, com $z=+1$. A maior estimativa do tempo de estocagem foi obtida quando se utilizou o teste acelerado em estufa, que parece ser mais realista visto que usa temperaturas mais próximas daquelas usualmente empregadas na estocagem de combustíveis.

Aplicando-se na equação conjunta os valores otimizados da concentração dos antioxidantes (Figuras 2 e 4), obtiveram-se os mesmos valores dos tempos de estocagem, isto é, 177,88 e 319,10 d quando se utilizou o Rancimat e o teste acelerado de estufa, respectivamente, mostrando que a equação, que inclui a variável de processo, pode ser usada para fins preditivos. 


\section{CONCLUSÃO}

O teste $\mathrm{t}$ aplicado aos parâmetros contendo a variável de processo mostrou diferença significativa entre o método Rancimat e o de estufa e, independentemente do antioxidante utilizado, todos os ensaios realizados com o teste acelerado em estufa apresentaram período de estocagem superior a 177,88 d, maior valor obtido pelo Rancimat, demonstrando que o método acelerado em estufa, o qual utiliza temperaturas que mais se assemelham às condições normais de estocagem, é o mais indicado para a determinação do tempo de armazenamento.

\section{MATERIAL SUPLEMENTAR}

As Figuras $1 \mathrm{~S}$ e $2 \mathrm{~S}$ mostram a região de contorno da resposta tempo de estocagem obtida com o método Rancimat e o método acelerado em estufa, respectivamente. A Tabela $1 \mathrm{~S}$ mostra os parâmetros de conformidade do biodiesel B100 de óleo de soja utilizado. Esse material encontra-se disponibilizado em http://quimicanova.sbq.org. br, na forma de arquivo PDF, com acesso gratuito.

\section{AGRADECIMENTOS}

Ao CNPQ pela concessão de bolsas e à UEL e Fundação Araucária pelo suporte financeiro.

\section{REFERÊNCIAS}

1. Lôbo, I. P.; Ferreira, S. L. C.; Cruz, R. S.; Quim. Nova 2009, 32, 1596.

2. Fukuda, H.; Kondo, A.; Noda, H.; J. Biosci. Bioeng. 2001, 92, 405.

3. Borsato, D.; Guedes, C. L. B.; Moreira, I.; Pinto, J. P.; Dias, G. H.; Spacino, K. R.; Semina 2010, 31, 3.

4. Thompson, D. R.; Transactions of the Assae 1981, 24, 1077.

5. Cornell, J. A.; Experiments with mixtures - designs, models and the analysis of mixture data, $2^{\text {nd }}$ ed., John Wiley and Sons: New York, 1990.

6. Marchi, J.; Bressiani, J. C.; Bressiani, A. H. A.; Bruns, R. E.; Int. J. Appl. Ceram. Technol. 2010, 7, 493.

7. Scheffé, H.; J. Royal Statistical Soc. B 1963, 25, 235.

8. Cornell, J. A.; Deng, J. C.; J. Food Sci. 1982, 47, 836.

9. Breitkreitz, M. C.; Jardim, I. C. S. F.; Bruns, R. E.; J. Chromatogr., A 2009, 1216, 1439.

10. Borsato, D.; Dall'Antonia, L. H.; Guedes, C. L. B.; Maia, E. C. R.; Freitas, H. R.; Moreira, I.; Spacino, K. R.; Quim. Nova 2010, 33, 1726.

11. Maia, E. C. R.; Borsato, D.; Moreira, I.; Spacino, K. R.; Rodrigues, P. R. P.; Gallina, A. L.; Fuel Process. Technol. 2011, 20, 1750.

12. Coscione, A. R.; Andrade, J. C.; May, G. M.; Quim. Nova 2005, 28, 1116.
13. Hare, L. B.; Technometrics 1979, 21, 159.

14. Anderson-Cook, C. M.; Goldfarb, H. B.; Borror, C. M.; Montgomery, D. C.; Canter, K. G.; Twist, J. N.; Pharmaceutical Statistics 2004, 3, 240.

15. Arteaga, G. E.; Li-Chan, E.; Vazquez-Arteaga, M. C.; Nakai, S.; Trends Food Sci. Technol. 1994, 5, 243.

16. Chen, J. S.; Lee, C. M.; Crapo, C.; J. Food Sci. 1993, 58, 535.

17. Derringer, G.; Suich, R.; J. Quality Technol. 1980, 12, 214.

18. European Committee for Standardization; EN 14112:2003 - Fat and Oil Derivatives - Fatty Acid Methyl Esters (FAME), Determination of oxidation stability (accelerated oxidation test), Berlin, 2003.

19. Frankel, E. N.; Trends Food Sci. Technol. 1993, 4, 220.

20. Antoniassi, R.; Bol. Centro Pesqui. Process. Aliment. 2001, 19, 353.

21. Ferrari, R. A.; Souza, W. L.; Quim. Nova 2009, 32, 106.

22. A.O.A. C. - Association of Official methods of Analysis; Official Methods of Analysis of AOAC International, $16^{\text {th }}$ ed., Washington, D.C., 1995, vol. 2.

23. Statistica For Windows v. 9.0; Tulsa, OK, USA, 2009.

24. Calado, V.; Montgomery, D.; Planejamento de experimentos usando o Statistica, E-Papers Serviços Editoriais: Rio de Janeiro, 2003.

25. ASTM International; ASTM D4052 - 09 Standard Test Method for Density, Relative Density, and API Gravity of Liquids by Digital Density Meter, 2002.

26. ASTM International; ASTM D93 - 10a Standard Test Methods for Flash Point by Pensky-Martens Closed Cup Tester, 2001.

27. ASTM International; ASTM D664 - 11 Standard Test Method for Acid Number of Petroleum Products by Potentiometric Titration, 2001.

28. ASTM International; ASTM D6584 - 10 Standard Test Method for Determination of Total Monoglyceride, Total Diglyceride, Total Triglyceride, and Free and Total Glycerin in B-100 Biodiesel Methyl Esters by Gas Chromatography, 2003.

29. EN 14110:2003; Fat and oil derivatives. Fatty Acid Methyl Esters (FAME). Determination of methanol content, 2003.

30. EN 14103:2003; Fat and oil derivatives. Fatty Acid Methyl Esters (FAME). Determination of ester and linolenic acid methyl esters content, 2003

31. EN 14214:2008; Automotive Fuels. Fatty Acid Methyl Esters (FAME) for diesel engines - Requirements and test methods, 2008

32. Karaosmanoglu, F.; Becker, U. G.; Energy Sources 1996, 18, 637.

33. Brasil, Agência Nacional do Petróleo, Gás Natural e Biocombustíveis; Resolução $n^{o} 7$, de 19/03/2008, estabelece a especificação para a comercialização de biodiesel que poderá ser adicionado ao óleo diesel, Diário Oficial da União, Brasília, 20/03/2008.

34. Frankel, E. N.; J. Sci. Food Agric. 1991, 54, 495.

35. Hasenhuettl, G.; Wan, P. J.; J. Am. Oil Chem. Soc 1992, 69, 525.

36. Xin, J.; Imahara, H.; Saka, S.; Fuel 2009, 88, 282. 


\section{COMPARAÇÃO DOS MÉTODOS DE DETERMINAÇÃO DA ESTABILIDADE OXIDATIVA DE BIODIESEL B100, EM MISTURA COM ANTIOXIDANTES SINTÉTICOS. APLICAÇÃO DO DELINEAMENTO SIMPLEX- CENTROIDE COM VARIÁVEL DE PROCESSO}

João Rafael de Moraes Cini, Dionísio Borsato*, Carmen Luísa Barbosa Guedes, Hágata Cremasco da Silva e Rodolfo Lopes Coppo

Departamento de Química, Universidade Estadual de Londrina, CP 6001, 86051-990 Londrina - PR, Brasil

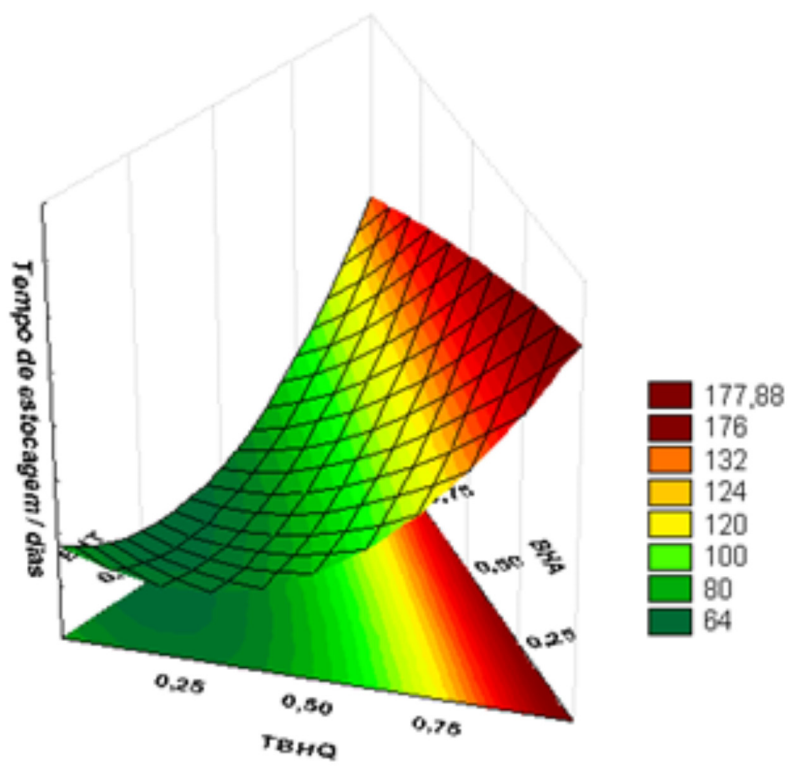

Figura 1S. Região de contorno da resposta tempo de estocagem obtida com o método Rancimat

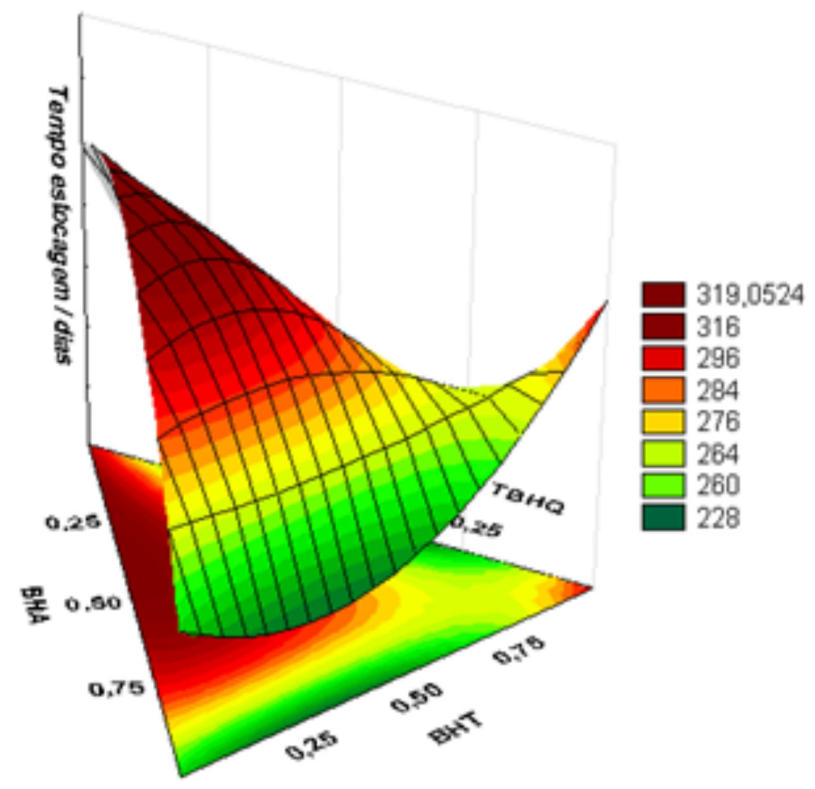

Figura 2S. Região de contorno da resposta tempo de estocagem obtida com o teste acelerado em estufa

Tabela 1S. Parâmetros de conformidade, de acordo com a especificação da ANP33, do biodiesel B100 de óleo de soja utilizado

\begin{tabular}{|c|c|c|c|c|}
\hline Característica & Método de ensaio & Unidade & Limite & Resultado \\
\hline Massa específica a $20^{\circ} \mathrm{C}$ & ASTM D4052 & $\mathrm{kg} \mathrm{m}^{-3}$ & $850-900$ & 881,7 \\
\hline Ponto de fulgor & ASTM D93 & ${ }^{\circ} \mathrm{C}$ & Min.100 & 160,0 \\
\hline Índice de acidez & ASTM D664 & $\mathrm{mg} \mathrm{KOH} \mathrm{g}^{-1}$ & Max. 0,50 & 0,40 \\
\hline Glicerina livre & ASTM D 6584 & $\%$ massa & Max. 0,02 & 0,002 \\
\hline Glicerina total & ASTM D 6584 & $\%$ massa & Max. 0,25 & 0,004 \\
\hline Monoglicerídeos & ASTM D6584 & $\%$ massa & Anotar & 0,01 \\
\hline Diglicerídeos & ASTM D6584 & $\%$ massa & Anotar & 0,025 \\
\hline Trigligerídeos & ASTM D6584 & $\%$ massa & Anotar & N.D. \\
\hline Metanol & EN 14110 & $\%$ massa & Max. 0,20 & N.D. \\
\hline Teor de éster & EN 14103 & $\%$ massa & Min. 97,28 & 99,86 \\
\hline
\end{tabular}

N.D.= Não Detectado 